\title{
El Instituto Geográfico y la cartografía catastral
}

Manuel-G. Alcázar Molina, Universidad de Jaén, D. G. Catastro, en excedencia; María Dolores Chica Palomo, Universidad de Jaén

De la mano de José Echegaray, y por Real Decreto de 12 de septiembre de 1870, se creó el Instituto Geográfico en la Dirección General de Estadistica del Ministerio de Fomento, siendo su primer director el coronel Ibáñez e lbáñez de lbero. Su misión era: "Realizar los trabajos relativos a la determinación de la forma y dimensiones de la Tierra, triangulaciones geodésicas de diversos órdenes, nivelaciones de precisión, triangulación topográfica, topografia del mapa y de catastro, así como las cuestiones relativas a pesos y medidas"2.

Echegaray promovió la actualización de los amillaramientos y responsabilizó al Instituto Geográfico (IG) de la realización de los deslindes de términos municipales y de los levantamientos planimétricos de las denominadas masas de cultivo ${ }^{3}$, que serian utilizadas en una futura aplicación catastral. Estos trabajos no soliviantaron a los grandes terratenientes, que no deseaban un catastro fiable que acabase con una situación crónica de ocultación de superficies, y se pudieron llevar a cabo sin oposición política.

En 1879 se elaboraron las Comparaciones de las superficies consignadas en el último amillaramiento oficial según los datos que existen en la D. G. de Contribuciones con las que resultan de los planos levantados por el Instituto Geográfico y Estadistico. Se trata de un documento imprescindible para comprobar objetivamente la situación rural de las provincias en las que se realizó: superficies, distribución de cultivos, calidades e, indirectamente, el posible grado de fraude fiscal existente.

A modo de ejemplo, en la provincia de Jaén, se comprueba la existencia de términos municipales en los que la ocultación de superficies alcanzaba cifras próximas al 400\%, y otros en los que las declaraciones de los propietarios superaban las superficies levantadas por el IG. El análisis detallado de esta situación daria lugar a un artículo independiente, pero baste decir en este texto que el afloramiento masivo de superficies, distribuidas por cultivos y calidades, favoreció la decisión de implantar un catastro gráfico nacional. La confección de un catastro topográfico-parcelario era ya sólo una cuestión de tiempo y de asignación de recursos (económicos y técnicos).

Aprovechando la cartografía mencionada, y siguiendo el ejemplo francés, el ministro de hacienda Navarro Reverter implantó ${ }^{4}$ el Catastro por masas de cultivo y clases de terreno ${ }^{5}$, que se generalizó en 1900, después de las pruebas piloto realizadas en varias provincias. Utilizando el mapa municipal elaborado por el IG (1:25.000), en el que se representaban los accidentes geográficos más importantes y las grandes masas de cultivo y clases de terreno, los responsables incluian en cada una de ellas las propiedades (sin representación parcelaria) declaradas por sus propietarios.

Los parámetros básicos en torno a los que giraba este nuevo proyecto legislativo fueron la elaboración de unos registros fiscales de la propiedad, uno por cada una de las riquezas contempladas (edificios y solares, rústica y ganaderia), que constituirían la base sobre la que gravarian los tipos impositivos para obtener las recaudaciones fiscales buscadas por el ejecutivo.

La cartografía catastral disponible no identificaba las parcelas/ fincas existentes gráficamente, pero sí las localiza dentro de su respectiva masa de cultivo. Con fines claramente fiscales permitió este catastro acotar el fraude en superficies, en titularidad y en calidades agronómicas. Desde un punto de vista histórico hay que resaltar que a partir de este momento se cuenta con mapas de cultivos y aprovechamientos, calificados en función de sus respectivas calidades (básicamente productividad), referenciados geográficamente y perfectamente superficiados. Todo ello favorecerá el conocimiento del país y fortaleció las estadísticas generales, tan demandadas durante todo el siglo XIX, en línea con los trabajos desarrollados en el resto de Europa.

Complementariamente, en el ámbito urbano, y a partir de 1893, comenzaron los trabajos para elaborar el Registro fiscal de edificios y solares, empleando para ello la cartografía urbana existente y elaborando croquis acotados de las edificaciones. De menor importancia tributaria y grado de ocultación, era imprescindible para conocer el patrimonio de los núcleos urbanos.

En 1902, nuevamente Echegaray, que preside la Junta de Catastro, impulsa la aprobación de la Ley de 23 de marzo de 1906, por la que se establecía el demandado Catastro Topográfico Parcelario. Un reflejo de su transcendencia se encuentra al comprobar su vigencia durante casi un siglo, y su refundición en el texto aprobado por RDL 1/2004.

El objetivo perseguido era la identificación individual, literal y gráfica, de todas las parcelas para su posterior evaluación y puesta en tributación. Se pretendía acabar con la desigualdad contributiva existente; emplear la documentación con fines estadisticos, sociales y económicos y, quizá lo más complicado, otorgar a este registro de 


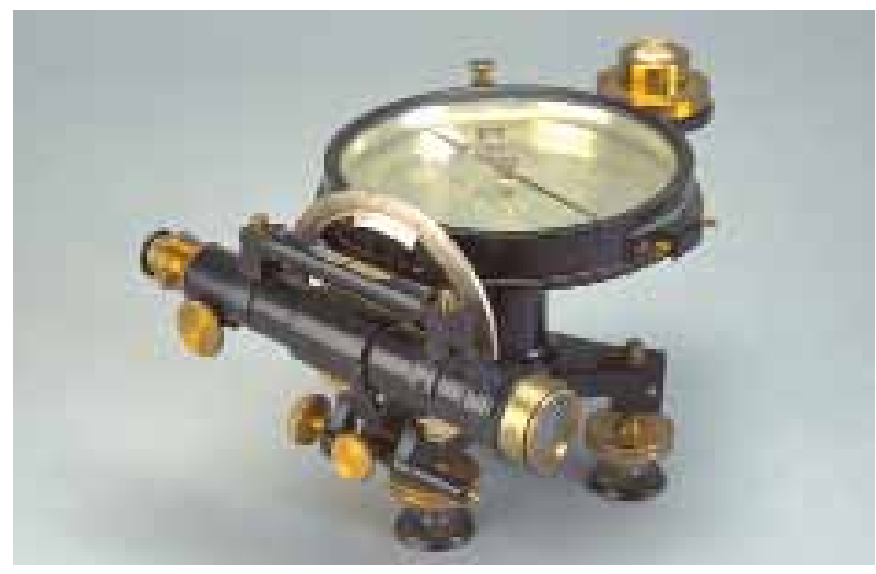

Brújula taquimétrica. Breithaupt\&Söhn. Hacia 1890. Fuente: Instituto Geográfico Nacional, ref. 183

bienes inmuebles la validez jurídica tan demandada y deseada por gran parte de la sociedad. Las dificultades económicas y la escasa disponibilidad de técnicos cualificados, así como la oposición de los grandes propietarios a su puesta en marcha, atrasaron ésta y no fue hasta 1913, con la aprobación del Reglamento de Avance Catastral Rústico, cuando comenzaron los trabajos distribuidos en dos etapas complementarias:

1. Avance. En primer lugar se realizaría un avance catastral que permitiese un reparto justo de la presión fiscal y un empleo simultáneo en aplicaciones estadísticas y administrativas, descubriendo superficies ocultas y evaluando correctamente la riqueza imponible ${ }^{6}$.

2. Conservación. Consistiría en la conservación y rectificación del Avance hasta conseguir el catastro topográfico-parcelario deseado, de acuerdo con el art. $2^{\circ}$ de la Ley: "El Catastro comprenderá en su conjunto la enumeración y descripción literal y gráfica de los predios rústicos y forestales, pertenencias mineras, solares, edificios, salinas, etc., con expresión de superficies, situación, linderos, cultivos o aprovechamientos, calidades, valores, beneficios y demás circunstancias que den a conocer la propiedad territorial y la definan en sus diferentes aspectos y aplicaciones".

Los trabajos topográficos, apoyados en la Red Geodésica Nacional, comenzaban con la captura de la información necesaria para la elaboración de un mapa a escala 1:25.000, conocido coloquialmente como "pañoleta", en el que se marcaban los poligonos catastrales junto con toda aquella información geográfica que se consideraba de interés: caminos, ríos, núcleos urbanos, diseminados importantes, toponimia... Simultáneamente, y con los datos capturados en campo, se elaboraban planos a escala 1:5.000 (opcionalmente 1:2.000 a 1:12.000 dependiendo de las características del terreno y del grado de parcelación) de todos y cada uno de los polígonos definidos.

Una vez decidido por el servicio de Catastro del Ministerio de Hacienda los municipios que iban a ser catastrados, se recibía del IG la documentación gráfica elaborada. Apoyándose en las declaraciones de los titulares, los técnicos identificaban las parcelas rústicas, los edificios y solares mediante un croquis, con ayuda de los prácticos, anotando las características físicas que permitan individualizarlos (situación, forma, linderos y extensión), identificándose el cultivo, si eran rústicas, o las caracteristicas constructivas, si eran urbanas. Esta información se enmarcaba dentro de accidentes geográficos, vías de comunicación, núcleos urbanos, construcciones aisladas, arroyos y ríos, parajes, etc.

Al igual que el Catastro de Ensenada, que fue una "foto fija" -principalmente literal- de la España de mediados del siglo XVIII, el Avance constituyó una representación croquizada (dentro de un marco geométrico preciso) de gran parte del país.

Posteriormente, y dentro ya del proceso de conservación, el IG topografiaba todas las parcelas catastrales, identificando los cultivos y calidades a través de las subparcelas. Esta actividad estuvo bajo la responsabilidad del IG hasta finales de los ochenta y convivió con la elaboración de catastros sobre fotografía aérea.

Las brigadas topográficas capturaban en campo los datos (generalmente con brújulas taquimétricas), y en gabinete elaboraban los denominados "catastrones". Sobre papel milimetrado iban representando las diferentes parcelas y subpacelas, sin olvidar la información geográfica. Obviamente este trabajo de campo era empleado, mediante una generalización posterior, para la elaboración del Mapa Topográfico Nacional, 1:50.000, producto emblemático del IG.

El resultado final eran unos planos, con intervalos de escala comprendidos entre 1:2.500 y 1:12.000 (mayoritariamente 1:5.000), de los municipios catastrados.

Posteriormente se sucedieron diversos proyectos catastrales: jurídico, masas de cultivo, fotografía aéreas... pero el IG continuó trabajando con los parámetros técnicos establecidos en los procesos de implantación y conservación diseñados en 1906. Por este motivo, la cartografía catastral de la Institución presenta una uniformidad que favorece todo tipo de estudios que tengan a la propiedad patrimonial inmobiliaria como elemento de referencia.

\section{Notas}

${ }^{1}$ A lo largo de su historia ha cambiado de nombre y de adscripción administrativa. ${ }^{2}$ Artículo $5 .{ }^{\circ}$

${ }^{3}$ Superficies rurales con aprovechamientos y rendimientos agrarios homogéneos de más de 10 ha.

${ }^{4}$ Formalmente podría hablarse de una "reimplantación", dado que esta metodología ya se habia probado con anterioridad en España.

${ }^{5}$ Ley de 24 de agosto y los Reales Decretos de 14 de septiembre y 29 de diciembre de 1896. Ley de 27 de marzo de 1900.

${ }^{6}$ Se preveía que este fase estuviese finalizada en quince años y, con la información capturada y los ingresos obtenidos por el aumento de la recaudación, se abordaría la segunda fase, la conservación, más costosa y lenta.

\section{Bibliografía}

ALCÁZAR MOLINA, M. (2007) Catastro Inmobiliario. Jaén: Caja Rural, 2007 GARCÍA-BADELL ABADÍA, G. (1942) El catastro de la riqueza rústica en España. Madrid: Ministerio de Agricultura, 1942 